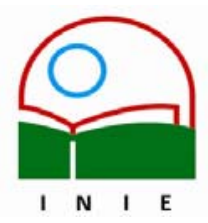

Universidad de Costa Rica

Facultad de Educación

Instituto de Investigación en Educación

ACTUALIDADES INVESTIGATIVAS EN EDUCACION

\title{
ACREDITACIÓN INTERNACIONAL: EL CASO DE LA AGENCIA DE ACREDITACIÓN CANADIENSE CCPE- CEAB EN LAS UNIVERSIDADES COSTARRICENSES
}

\author{
Mónica Quirós Villalobos ${ }^{1}$ \\ Rocío Arce Durán ${ }^{2}$
}

\begin{abstract}
RESUMEN: Las tendencias mundiales han motivado a las instituciones de educación superior a replantear su labor académica y el perfil profesional con el que sus estudiantes inician su práctica profesional, en búsqueda de una mayor calidad. La necesidad de que las universidades garanticen a la sociedad profesionales de alta calidad, con capacidad para desempeñarse, tanto en el contexto nacional como en el internacional, ha propuesto que algunas carreras utilicen estándares internacionales para la evaluación de sus programas.

El propósito de este artículo es presentar algunos de los resultados de la investigación denominada, "Contextualización de los sistemas de acreditación internacional: el caso de la agencia de acreditación canadiense CCPE-CEAB en las universidades costarricenses", realizada en el marco del curso CAMINA-Costa Rica 2002. En esta investigación, se analizó cómo el Sistema de Acreditación Canadiense CEAB, especializado en ingeniería, ha influido en algunos de los programas de Ingeniería de distintas universidades de Costa Rica.
\end{abstract}

Palabras claves: ACREDITACION INTERNACIONAL DE PROGRAMAS DE INGENIERÍA COSTARRICENSE/ AGENCIA CANADIENSE DE ACREDITACION/

\begin{abstract}
The world's trends have motivated the college level institutions to rearrange their academic activities, and to review the student's profile regarding their professional practices, in search of improvement. The need for universities vouching to society high-quality professionals, professionals that capable of performing adequately in national and international contexts, has made some careers to seek evaluations by international standards of their programs.

The purpose of this article is to present some of the results of the project named "Contextualization of the international certification systems: the case of the Canadian CCPE-CEAB certification agency in Costa Rican universities", which was carried out within the framework of the Camina-2000 course. In this investigation, the manner in which Canadian CEAB Certification System has influenced some of the engineering programs in several Costa Rica's universities was analyzed.
\end{abstract}

Key words: ACCREDITATION THE INTERNATIONAL OF PROGRAMS OF COSTA RICAN ENGINEERING/ CANADIAN AGENCY OF ACCREDITATION/

\begin{abstract}
${ }^{1}$ Egresada del Plan de Estudios de la Maestría en Comunicación Colectiva, Egresada del Plan de Estudios de la Licenciatura en Antropología Social, Bachiller en Antropología Social, todos los títulos de la Universidad de Costa Rica. Actualmente labora en el Centro de Evaluación Académica, en el Depto. de Investigación y Evaluación Académica como investigadora docente. Labora también como investigadora en el Centro de Investigación en Estudios de la Mujer. Correo electrónico mquirosv@cariari.ucr.ac.cr

2 Maestría en Evaluación de Programas y Proyectos Sociales, Licenciatura en Sociología, ambos de la Universidad de Costa Rica. Actualmente labora en el Centro de Investigación y Evaluación Institucional donde realiza labores de investigación y evaluación, de la Universidad Estatal a Distancia (UNED). Además es docente de la carrera de Administración de Empresas, impartiendo el curso de investigación dirigida de la última universidad. Correo electrónico: marced@uned.ac.cr
\end{abstract}

Artículo recibido: 14 de julio, 2005

Aprobado: 26 de setiembre, 2005 


\section{Introducción}

Este artículo presenta los resultados obtenidos en la investigación mencionada en el resumen y que se realizó en el curso de capacitación CAMINA-COSTA RICA 2002, y que tiene como antecedente el aprendizaje realizado en Alemania, Costa Rica y Guatemala, entre 1999 y el 2001. En este proceso, participaron académicos y académicas de las diferentes universidades de la región centroamericana. Esta actividad fue organizada por el Consejo Superior Universitario Centroamericano (CSUCA) con el apoyo de la Fundación Alemana para el Desarrollo Internacional y el Instituto de Estudios Socioculturales de la Universidad de Kassel. La experiencia de este grupo de profesionales capacitados, promovió el curso CAMINA-COSTA RICA 2002, cuyos objetivos fundamentales eran apoyar a las universidades costarricenses en el mejoramiento de la calidad de la educación y generar espacios de discusión y redes de apoyo, entre los equipos de evaluación de diversas instituciones educativas privadas y públicas, en torno a la autoevaluación y la acreditación.

Como parte de esta capacitación, se elaboraron varias investigaciones en distintas temáticas. Una de ellas fue la acreditación internacional, en donde se inscribe la investigación que muestra el presente artículo.

Para disponer una mayor comprensión de la terminología, es importante tener presente, antes de exponer los resultados de la investigación, que el CEAB constituye una agencia de acreditación canadiense, la cual brinda a los programas académicos de su país, la acreditación y fuera de este, ofrece una equivalencia sustancial. Por tanto, es fundamental recordar que los programas de ingeniería que han cumplido con los criterios mínimos de la agencia, cuentan con una equivalencia sustancial de su plan de estudios con respecto a los programas académicos.

La interrogante que condujo esta investigación fue la siguiente: ¿De qué manera el sistema de acreditación canadiense especializado en Ingeniería (CCPE-CEAB), ha influido en los programas de ingeniería de las carreras que han obtenido la equivalencia sustancial o en proceso de visita de pares externos $?^{2}$ Se ofreció una primera aproximación de estos

\footnotetext{
${ }^{2}$ Es importante tener presente que, durante la elaboración de la investigación, se encontraban varios programas de ingeniería que esperaban la visita de los pares externos. Por este motivo, se les consideró en la muestra, Volumen 5, Número Especial, Año 2005, ISSN 1409-4703 
procesos de internacionalización y homogenización en los programas de ingeniería costarricense.

Esta investigación presenta un estudio descriptivo, exploratorio y analítico. Los ejes de análisis fueron los siguientes:

- Contextualización de la equivalencia sustancial en los programas de ingeniería de las universidades estatales: condiciones previas, objetivos de la acreditación y propósitos.

- Efectos del modelo y los criterios evaluativos del CEAB en los programas de ingeniería que han obtenido la equivalencia sustancial o están en proceso de la visita de pares externos de esta agencia.

Para el primer eje de estudio, fue necesario realizar una contextualización de los modelos canadienses de acreditación, especializados en la rama de las Ingenierías que permitiera identificar aspectos tales como: antecedentes, momento educativo histórico al que responde y el perfil profesional de la educación superior.

El análisis del modelo educativo propuesto por la agencia de acreditación canadiense, se realizó partiendo de las experiencias de la Universidad de Costa Rica, la Universidad Nacional, el Instituto Tecnológico de Costa Rica y la Universidad Latina. El estudio se efectuó por medio de una revisión de contenido y de aspectos como: conceptos empleados, criterios y lineamientos, perfil profesional y visión general de la disciplina.

El segundo eje se realizó por medio de un estudio de casos que incluyó a los programas de ingeniería de las universidades estatales y privadas, cuyos programas habían adquirido la equivalencia sustancial o estaban en el proceso de solicitud y visita de pares externos de la agencia CEAB en el momento de la investigación. Se presentaron los siguientes:

- Ingeniería Eléctrica de la Universidad de Costa Rica.

- Topografía de la Universidad Nacional.

- Ingeniería en Electrónica de la Universidad Latina.

- Ingeniería en Construcción del Instituto Tecnológico de Costa Rica.

- Ingeniería de Producción Industrial del Instituto Tecnológico de Costa Rica.

Igualmente se contó con la colaboración del Colegio Federado de Ingenieros y Arquitectos (CFIA), específicamente del Consejo de Acreditación presidida por el ingeniero Roberto Trejos Dent. 


\section{Antecedentes: la acreditación en el contexto internacional}

La economía mundial y sus principales tendencias, han propiciado la internacionalización de los procesos productivos, la integración de los mercados financieros y la conformación de espacios comerciales regionales.

En este sentido, el sector educativo cumple un papel fundamental, pues su rol en la generación de los conocimientos y formación de recursos humanos, lo lleva a garantizar, por una parte, los conocimientos y habilidades básicas de la fuerza laboral, facilitando su capacitación para el trabajo y su incorporación en empleos productivos y, por otra, la base de profesionales y científicos que alientan la innovación y la generación de fuentes endógenas de crecimiento económico y social.

Las diversas políticas de integración económica, como los tratados de libre comercio con el Norte, han traído cambios significativos en todos los ámbitos de las relaciones económicas y sociales de la Región Centroamericana. Como medida estratégica, los países han venido reforzando sus ventajas competitivas, fortaleciendo los sistemas educativos que se han visto en la necesidad de una modernización permanente; en otras palabras,

adecuarse a las nuevas condiciones y retos, por lo que el establecimiento de sistemas de certificación y acreditación que agilicen la movilidad de profesionales en condiciones de competencia equitativa y el impulso a líneas de investigación prioritarias para el desarrollo nacional son asuntos fundamentales en donde la participación de la educación superior será de gran envergadura. (Gómez, 2001, p.7)

Esta participación se centra, principalmente, en la oferta de los servicios profesionales, en donde se requiere que cada país reconozca los títulos expedidos en los institutos de Educación Superior de los países socios (miembros de los convenios) y la autorización en el ejercicio profesional de extranjeros en sus respectivos territorios.

De esta manera, se buscan sistemas de acreditación internacional

...homogéneos para la expedición de licencias y certificados a profesionales y para la acreditación de instituciones y programas de estudio que garanticen egresados de calidad estándar a lo largo de la macroregión. Naturalmente, este proceso requiere 
que los contenidos de estudio y los sistemas de evaluación a través de los cuales se realiza la acreditación sean también más homogéneos. (Gómez, 2001, p. 8)

Como resultado de lo anterior, la calidad de las instituciones de educación superior y la internacionalización de sus graduados, han promovido la formación de un sistema de acreditación regional que certifica las destrezas y los conocimientos individuales de los profesionales, a través de procesos de autoevaluación y autorregulación de la calidad académica de las instituciones, de sus planes y programas de estudio. Esta labor reconoce que todo proceso de acreditación institucional, debe considerar los objetivos educativos de la institución; su capacidad de dotación de recursos materiales, humanos y financieros; su gestión y eficiencia administrativa.

Las tendencias internacionales exigen la consolidación de grandes esfuerzos entre los países y sus respectivas instituciones de la enseñanza superior, para elevar la calidad de sus procesos educativos y sentar las bases para la construcción de sistemas de acreditación. De esta forma, Estados Unidos y Canadá tienen una ventaja considerable sobre los países de América Latina, ya que ambos países han desarrollado consolidados procesos sistemáticos de acreditación.

En el caso de los Estados Unidos, el sistema de acreditación presenta sólidas bases. Su organización se basa en las asociaciones regionales privadas y voluntarias de las universidades y los colegios universitarios, donde participan profesionales y académicos de reconocido prestigio. El sistema exige una serie de requisitos concretos a las instituciones solicitantes, y realiza procesos de autoevaluación y de visita de pares. La clave del éxito ha sido la importancia dada a la autoevaluación institucional, pues es más fácil que cada institución detecte deficiencias integrales y emprenda las acciones de autorregulación apropiadas, en lugar de las instancias ajenas.

En Canadá, aunque cuentan con un sistema de acreditación, que es distinto del que funciona en los Estados Unidos, ha tenido un fuerte impacto en lo que denominan sistema de Educación Superior (SES). Este sistema se implementa desde el sector público, sometiendo, obligatoriamente, a todas las instituciones y programas de los procesos de acreditación. 


\section{La Agencia Canadiense de Acreditación en Ingeniería - CEAB}

Como agencia acreditadora de programas de ingeniería, la Canadian Engineering Accreditation Board (CEAB) certifica tanto en el nivel nacional como internacional. Esta agencia forma parte del Consejo Canadiense de Ingenieros e Ingenieras Profesionales (CCPE), establecido en 1963, como la Federación de Autoridades Provinciales y Territoriales de Canadá para extender licencias a las personas ingenieras y supervisar la profesión en todo el país. Sus principales acciones son acreditar los programas de grado en ingeniería y velar para que se cumplan los estándares educativos aceptables de la profesión en su país. Asimismo, se encarga de establecer la equivalencia de sistemas de acreditación en otros países y supervisar las actividades de estos entes con los que se hayan firmado acuerdos de reconocimiento.

Puntualmente, sus objetivos son los siguientes:

1. Acreditar los programas de grado de ingeniería en Canadá y asegurar que reúnan los estándares aceptables de educación, para que se registren como programas profesionales de ingeniería en las provincias y territorios de Canadá.

2. Certificar la equivalencia y aceptabilidad de los sistemas de acreditación en otros países y negociar acuerdos de reconocimientos bilaterales con otros organismos que cuenten con sistemas aceptables para el CCPE.

3. Aconsejar y cooperar con el CCPE en los asuntos relacionados con la educación en ingeniería.

4. Evaluar programas de ingeniería en otros países de acuerdo con los criterios y políticas de la CEAB, otorgándoles una equivalencia sustancial. La institución interesada asume el costo.

En general, la $C E A B$ valora al programa por el número de unidades de acreditación, las cuales se definen como: una hora de clase que equivale a una unidad de acreditación y una hora de laboratorio o tutoría que equivale a 0.5 unidades de acreditación, y se enfatiza la evaluación cualitativa del programa y el contexto en el que está inmerso, mediante un instrumento cuantitativo muy preciso y exhaustivo, ya que la CEAB sostiene que el instrumento cuantitativo debe visualizarse, como un proceso cualitativo del análisis e investigación del programa académico. 
Siendo los ejes curriculares los componentes evaluativos, los criterios evaluativos centrados en las unidades de acreditación, se citan a continuación:

- Matemáticas: un mínimo de 195 unidades de acreditación, las cuales deben incluir una serie de materias relacionadas.

- Ciencias básicas: un mínimo de 225 unidades, en donde se incluyen la física, la química y los elementos de las ciencias de la vida y la tierra.

- Ciencias y diseño de ingeniería: el mínimo son de 900 unidades de acreditación en una combinación de ciencias y diseño de la ingeniería.

- Estudios complementarios: se requiere un mínimo de 225 unidades de acreditación en estudios de humanidades, ciencias sociales, artes, administración, economía de la ingeniería y comunicación.

El plan de estudios debe contener un mínimo de 1800 unidades de acreditación, y debe garantizar que sus estudiantes tomen conciencia del papel y la responsabilidad de los ingenieros e ingenieras dentro de la sociedad.

Es importante tener presente que la CEAB acredita $u$ otorga equivalencias sustanciales a los programas que cumplan con un mínimo de 16 años de escolaridad: 6 de primaria, 6 de secundaria y 4 de universidad. Para el caso de Costa Rica, el sistema educativo consta de 6 años de primaria, 5 de secundaria y 4 de bachillerato universitario, lo que da un total de 15 años. Con base en los programas de ingeniería que optaron por una equivalencia sustancial con los programas canadienses, se considera la licenciatura de su plan de estudios, como un año más para completar los 16 años de escolaridad mínimos que la CEAB establece.

\section{Los procesos de acreditación de las ingenierías en Costa Rica}

Con la participación de las principales agencias de acreditación especializadas en ingeniería de Norteamérica en 1989, se firma el Acuerdo Multinacional de Washington. En éste se define el reconocimiento o la equivalencia substancial de los sistemas de acreditación de las organizaciones que realizan los estados signatarios y los programas de la educación de la ingeniería acreditados por ellos. De esta manera, las personas graduadas de dichos programas académicos acreditados o con equivalencias sustanciales, tendrían la posibilidad de laborar en algún país miembro. Se debe tener en cuenta que cada país y/o estado tiene regulaciones específicas para autorizar el ejercicio de la profesión. Lo anterior implicaría 
certificar años de experiencia, presentar exámenes especializados y todos los requisitos solicitados por el país, al cual un profesional en ingeniería, quiera movilizarse para trabajar.

Visualizando las oportunidades internacionales para los ingenieros y las ingenieras nacionales, el Colegio Federado de Ingenieros y Arquitectos de Costa Rica (CFIA) inicia una serie de negociaciones con las agencias ABET (Accreditation Board for Engineering and Technology de Estados Unidos) y la CEAB (Canadá) con el propósito de iniciar los procesos de equivalencias sustanciales "especializados" en el país; capacitarse adecuadamente sobre el tema y, posteriormente, considerar un sistema de acreditación nacional propio ${ }^{3}$. De las dos agencias, se logra desarrollar un convenio con la CEAB y en 1999, se inician los procesos, considerando que el colegio sería quien asuma los costos, con previo estudio, de los programas de ingeniería de las universidades estatales y privadas que deseen optar por dicho modelo.

Los objetivos del colegio con respecto a este convenio de cooperación, en ese momento eran lograr la internacionalización de la ingeniería, permitiendo que aquel que posea un título universitario de una carrera "con equivalencia sustancial" por la CEAB pueda laborar en otro país, sin realizar gestión alguna para demostrar su calidad profesional (Coto, J, 1997, p. 7); garantizar el cumplimiento de todos los requisitos mínimos para el ejercicio profesional de los graduados y el reconocimiento profesional en la suscripción a convenios internacionales de reconocimiento mutuo entre los países interesados. Esto sin considerar que dichos procesos, promueven la calidad y el mejoramiento permanente de las carreras de ingeniería en el país. A partir del convenio con la $C E A B$, se realizan una serie de visitas a otros países y procesos de capacitación; se crea la Comisión de Acreditación y Licenciación en el CFIA con el propósito de establecer las primeras bases para el funcionamiento de un Consejo de Acreditación y se redactan los lineamientos operativos del consejo, estableciendo que: " $L a$ universidad que lo desee podrá solicitar que se acredite su programa, el Consejo de Acreditación entregará a las autoridades universitarias un cuestionario de autoevaluación y verificará los datos suministrados, los cuales serán la base para conceder o no la acreditación". (Coto, 1997, p.7).

\footnotetext{
${ }^{3}$ En el Seminario Centroamericano de Acreditación de las Ingenierías celebrado en Costa Rica en el 2004, el CFIA presentó una propuesta de manual de acreditación ante los participantes. 
En otras palabras, los programas deben ser revisados por el criterio del CFIA, antes de enviar la solicitud ante la CEAB. De esto dependerá el financiamiento para optar por la visita de pares externos y obtener la equivalencia sustancial.

Mientras el sistema nacional de acreditación en Ingenierías ${ }^{4}$, es puesto en práctica, muchos programas académicos en el área de la Ingeniería se han sometido a los criterios evaluativos del CEAB, optando por una equivalencia sustancial, lo que significa:

- Los programas son comparables en cuanto al plan de estudios y experiencia educativa.

- Existe confiabilidad en que las personas graduadas cuenten con la capacidad académica necesaria para comenzar la práctica profesional.

- Esta equivalencia representa el primer paso para un acuerdo bilateral.

Por otra parte, el convenio de colaboración con la CEAB ha permitido al CFIA realizar actividades tales como:

- Capacitación y pasantías en Canadá, Estados Unidos y México de profesionales del Colegio.

- Participar como observadores en las visitas de evaluadores de la CEAB.

- El desarrollo de un sistema de acreditación especializado que tenga como objetivo identificar, según criterios internacionalmente reconocidos, los programas de enseñanza de la ingeniería y de la arquitectura en los que los egresados han sido capacitados para la práctica moderna de la profesión (Coto, J, 1997, p. 7).

\section{Correspondencia de los objetivos, criterios y procedimientos del CCPE- CEAB con respecto al perfil de ingeniería de los programas académicos}

Cualquier modelo de acreditación de una u otra agencia acreditadota, propone una visión general de las condiciones, orientaciones, características y resultados de un programa académico. En este sentido, la elección de una agencia y sus criterios implican que el programa académico, de una u otra forma, asumirá la visión académica y profesional de la agencia seleccionada.

\footnotetext{
${ }^{4}$ Desde un inicio, el CFIA ha mostrado su interés por formar su propia agencia de acreditación. En este sentido, el considerar los criterios de la CEAB e iniciar las experiencias con las universidades constituyeron la oportunidad de ir adquiriendo experiencia en el tema de acreditación. Actualmente, el CFIA está estrechamente vinculado con la agencia nacional de acreditación SINAES.
} 
Muchas veces esta visión puede estar implícita o explícita en los criterios. En el caso de la $C E A B$, la visión del profesional en el área de ingeniería, debe leerse a partir de sus componentes y criterios, donde se establecen los rasgos generales de la ingeniería, desde una perspectiva integral y no solo por especialidad.

El manual de la CEAB parte de que los profesionales del área, deben tener una buena competencia en la ingeniería y comprensión del efecto de esta en la sociedad, lo que significa que los programas de ingeniería deben

Desarrollar la capacidad de los estudiantes para usar apropiadamente el conocimiento y la información para convertir, utilizar y administrar los recursos de manera óptima a través de un análisis efectivo, la interpretación y la toma de decisiones. Esta habilidad para el proceso de diseño que caracteriza la práctica de la ingeniería. (Municio, 1997, p. 23)

En otras palabras, exigen un profesional en el área que cuente con una visión integral de la profesión, no tanto desde una perspectiva de especialización, sino que comprenda el efecto de sus acciones profesionales en la sociedad, y que logre una lectura de las condiciones, personas y situaciones que rodean su labor profesional. Asimismo, se centra en la formación de profesionales orientados para tomar decisiones eficientes y eficaces, a partir de un proceso de análisis, interpretación y administración de la información y el conocimiento adquirido. Lo que significa una permanente relación entre la teoría y práctica.

El contenido curricular del manual ofrece mayores luces al respecto, ya que parte de que los programas cuenten con bases sólidas en matemática y ciencias básicas; una preparación amplia en las ciencias y diseño de la ingeniería y en cursos no técnicos que complementen los aspectos técnicos del currículum. (Municio, P, 1997, p. 25)

En el caso de la ingeniería en Costa Rica, el CFIA señala que cada programa de ingeniería posee un perfil específico, por lo que es difícil establecer los cambios que los programas con equivalencia sustancial del CEAB han generado en su currículum. Sin embargo, de acuerdo con los representantes del CFIA, la propuesta de la CEAB, en cuanto al perfil, no varía de los planteamientos curriculares de los programas académicos que se ofrecen en el país, ya que se plantea un ingeniero o una ingeniera con las siguientes características:

- Una visión internacional de su profesión, siendo capaz de desempeñarse en cualquier entorno y país.

- Ser capaz de formular y resolver problemas complejos referidos a su campo profesional. 
- Comprender y resolver las repercusiones ambientales, económicas y sociales de sus obras.

- Ser capaz de comunicarse efectivamente; trabajar en equipo; aprender en forma autónoma y evaluar, efectivamente, los efectos ambientales y sociales de las obras de ingeniería y arquitectura.

- Desarrollar y aplicar valores éticos y sociales.

- Reforzar la capacidad del diseño técnico.

- Desempeñarse apropiadamente en la sociedad contemporánea.

- Participar en programas de educación continua y de desarrollo profesional.

Como se puede apreciar, no existen diferencias significativas con la propuesta del manual del $C E A B$, excepto por el nivel de especialización, ya que igualmente se plantea un profesional integral, capaz de comprender y resolver problemas específicos de su entorno; que esté actualizado y con capacidad de trabajar en equipos multidisciplinarios. Igualmente parten de una concepción sustentada en la aplicación de la matemática y de las ciencias naturales para el diseño y desarrollo de dispositivos, estructuras y procesos utilizables, con criterios de eficiencia económica, con preocupación en el medio ambiente y para beneficio de la humanidad (Municio, P, 1997, p. 25). Es importante visualizar, además, un interés por el acervo conceptual e instrumental de las ciencias sociales en la disciplina.

En este sentido, a pesar de las similitudes en cuanto a la propuesta curricular de la CEAB y del CFIA, los empleadores manifiestan que los ingenieros y las ingenieras deben:

- Contar con la especialidad en ingeniería y tener conocimientos generales, tanto de la ingeniería, como de otras disciplinas; por ejemplo: administración y economía.

- Estar actualizados con el propósito de responder a las necesidades del mercado laboral nacional e internacional.

- Tener iniciativa y liderazgo.

- Manifestar habilidades de comunicación oral, escrita y gráfica.

- Encontrar soluciones a los problemas específicos y trabajar en equipo y bajo situaciones de presión.

- Dominar el inglés.

- Disponer de un razonamiento lógico y analítico, así como la creatividad en su desempeño laboral. 
Tanto la perspectiva de los empleadores, como de la CEAB y CFIA, coinciden en el perfil profesional de la persona ingeniera, resaltando además, la importancia del autoaprendizaje del profesional. Aunque, existen importantes coincidencias, el perfil costarricense muestra algunas diferencias significativas, importantes de hacer mención. Por ejemplo, que los graduados y las graduadas cuenten con un conocimiento especializado y con un fuerte tecnicismo, según las áreas de ingeniería que opten frente a una visión integral del CEAB. Asimismo, la CEAB recomienda a los programas, la capacidad de incluir habilidades, tales como: comunicación, interacción con otras disciplinas, incluso de sus propias áreas y la consideración de los enfoque sociales para su desempeño profesional.

Aunque es difícil establecer los posibles cambios o impactos del perfil por la agencia, los programas acreditados por la $\mathrm{CEAB}$, indican modificaciones en términos generales, centrados en la integración de conocimientos, habilidades, destrezas y valores propios de la persona ingeniera y la incorporación de contenidos del área social en su currículum.

\section{Condiciones que mediaron en la decisión de obtener la equivalencia sustancial con la agencia canadiense CEAB - CPPE}

La escogencia de una agencia de acreditación debe considerar, en primera instancia, los objetivos y la misión de la institución que la promueve, así como los fines estratégicos y académicos de los mismos, ya que cada agencia dentro de su manual, lleva implícito una forma de analizar, comprender y enseñar la disciplina. De esta forma, el proceso de decisión o selección debe llevar un análisis minucioso del planteamiento curricular determinado por la agencia.

En el caso del CFIA y CEAB, la escogencia de la agencia para optar por una equivalencia sustancial fue un proceso voluntario para las universidades y sugirió una etapa previa de análisis de cada uno de los criterios evaluativos. Por este motivo, la CEAB garantizaba el proceso y el CFIA los recursos tanto económicos como logísticos para llevar a cabo el proceso. Una de las razones que medió en la decisión de optar por la agencia canadiense y no por otra agencia fue que "...específicamente en el área de las ingenierías el CFIA empezó a estimular a las universidades públicas y privadas para que iniciaran este proceso y a través de una agencia de carácter internacional (Arce y Quirós, 2005a). 
Las razones aducidas por los profesionales de este Colegio Profesional, se orientaron en tres principales líneas de interés institucional. Primero, debido al problema que se había presentado por la proliferación de universidades privadas, les interesaba establecer estándares igualitarios para las universidades públicas y privadas.

Segundo, el proceso pudo haberse realizado por medio del SINAES que es una agencia nacional; pero al colegio le interesaba rescatar la parte internacional, ya que es muy importante la internacionalización de la ingeniería, en función de los procesos de globalización e, inclusive, porque constituye una de las áreas donde hay un intercambio mayor de profesionales a otros países. Tal vez en otras áreas, esta no sea la situación, pero en la ingeniería, las empresas y los profesionales de un país empiezan a ser contratados y pueden trabajar en otros países. Por lo tanto, hay una gran necesidad de que los profesionales estén estandarizados en su preparación académica, con respecto a los profesionales de otros países. La tercera línea del colegio se fundamenta en la instauración de un examen de incorporación y ejercicio de la profesión.

Otra de las condiciones que mediaron en participar con una agencia internacional, ha sido la transformación que ha ido sufriendo el mercado de trabajo para los nuevos profesionales, ya que con el fenómeno de la globalización, crecen las posibilidades y oportunidades de trabajar fuera del país, en donde se adquirió la profesión. Lo anterior, aunado al contexto internacional y los convenios de Libre Comercio - pero sobretodo con el Acuerdo Multinacional de Washington - el CFIA, se ha planteado como uno de sus objetivos: lograr la internacionalización de la Ingeniería, permitiendo que aquel que posea un título universitario de una carrera con equivalencia sustancial, pueda laborar en otro país sin realizar gestión alguna para demostrar su calidad profesional:

La posibilidad de ofrecer a sus estudiantes una carrera de carácter internacional, que les permita ser más competitivos en el mercado, que les asegurara los niveles de excelencia académica y los posibilitará a entrar precisamente de los procesos de globalización y poder participar eficientemente de todo el proceso que se da de intercambio de profesionales y poder trabajar con empresas extranjeras y ofrecer una alternativa real a su potencial desarrollo. (Arce y Quirós, 2005a)

La equivalencia sustancial con la agencia canadiense, CEAB, figura como una ventaja competitiva, tanto para los profesionales en ingeniería que se egresen de un programa que 
ha obtenido la equivalencia sustancial, como para la universidad a la cual pertenece este programa, ya que ofrece

La posibilidad de que los estudiantes que se gradúen a partir de la acreditación tengan la oportunidad de trabajar en Canadá, es decir, ser reconocidos. Básicamente, prestigio, un cierto prestigio para la escuela por tanta competencia que hay ahora; por consiguiente hay que buscar ciertas calificaciones que lo acrediten y que digan que es un programa realmente serio (...) Para los estudiantes es importante saber para qué les sirve la acreditación, que ventajas tienen de que el programa este acreditado. La ventaja es que pueden estar seguros de que les enseñaron en un programa que está avalado por un ente acreditador, que el programa es similar a universidades de países desarrollados, que le sirve para Canadá si lo desea y que pueden decir que se graduaron de una universidad que tienen un programa equivalente a las universidades canadienses y se lo pueden reconocer para trabajar. (Arce y Quirós, 2005a)

\section{Logros y limitaciones: la equivalencia sustancial con la agencia canadiense CEAB}

El presente apartado ofrece una visión general de los logros y limitaciones de este tipo de equivalencia sustancial, con el propósito de apoyar y fortalecer los futuros procesos, tanto nacionales como regionales.

\subsection{Logros de la equivalencia sustancial con el CEAB}

La equivalencia sustancial otorgada por la CEAB, como una experiencia nueva para los programas de ingeniería, no solo ha tenido efectos en su plan de estudios, sino también, en su concepción e importancia en torno a los temas de mejoramiento continuo y calidad académica. Como bien lo establece el Ing. Roberto Trejos, los programas de ingeniería “...tienen una responsabilidad muy grande y un prestigio que debe mantenerse" (Arce y Quirós, 2005d).

Los programas académicos de Ingeniería han comprendido la importancia de que independientemente de acreditación o equivalencia sustancial, constituye una herramienta para alcanzar la calidad académica y el mejoramiento permanente. 
Uno de los alcances más importantes, según los distintos programas consultados, es que la $\mathrm{CEAB}$, ofrece la posibilidad de que la población estudiantil de los programas de ingeniería del país, pueda competir internacionalmente con otros profesionales. Al respecto el Ing. Olman Vargas, Decano de la Facultad de Ingeniería de la Universidad Latina, señala que es importante, "ofrecer a sus estudiantes una carrera de carácter internacional, que les permita ser más competitivos en el mercado (...) y les posibilitará entrar precisamente en los procesos de globalización" (Arce y Quirós, 2005b).

Tanto para el Ing. Trejos, como el Ing. Olman Vargas, la equivalencia sustancial plantea la posibilidad de profesionales en ingeniería con un perfil internacional. Esto significa que las personas egresadas cuentan con la posibilidad de ejercer su profesión en otros países, generando nuevas oportunidades de empleo y desarrollo profesional, especialmente en Canadá, donde sus títulos y plan de estudios son "sustancialmente equivalentes". Esto está relacionado directamente, con el hecho de que para los programas de ingeniería estudiados, esta "equivalencia" permite determinar que ellos cumplen con una serie de requisitos que garantizan la calidad de su quehacer académico y autorregularse en forma permanente. Sin embargo, es importante tener presente que la movilidad profesional podría ser consecuencia de estos procesos.

En forma general, los efectos positivos de la equivalencia sustancial otorgada por la CEAB, están centrados en el mejoramiento de la calidad académica de los programas, respondiendo a los requerimientos de un entorno profesional más homogéneo, generalista e internacional.

Igualmente ha facilitado a lo interno de los programas, una visión sobre la importancia del mejoramiento constante y permanente, que les permitan retroalimentarse y tener una mayor conciencia de la labor académica. Al respecto, el Decano de la Facultad de Ingeniería de la Universidad de Costa Rica en el 2002, señala que ha permitido que los programas “...conozcan internamente cuáles son sus debilidades" (Arce y Quirós, 2005c); por ejemplo, su tendencia a aislarse evitando la comunicación con otras instancias universitarias; este es uno de los aportes más importantes, ya que

cada uno de los programas tiene que mantener una transferencia de servicios, de estructura de apoyo con las otras escuelas de la facultad, de la universidad y las 
autoridades; porque la universidad es una plataforma de servicios que todos tienen que apoyar (Arce y Quirós, 2005c).

En cuanto a las condiciones internas del plan de estudios, la mayoría de las personas entrevistadas afirmó que los cambios no han sido radicales, exceptuando algunas carreras; pero sí indican que se ha mejorado, significativamente, el plan de estudios y, sobre todo, la concepción del profesional en ingeniería. Dentro de los efectos positivos, la investigación reveló que:

- Se logró determinar que los programas de ingeniería del país, superan los requisitos mínimos solicitados por el manual del CEAB, especialmente en cuanto a las unidades de acreditación de los cursos. A pesar de que no se ajustaban al modelo educativo canadiense, donde la secundaria incluye cursos que normalmente un estudiante costarricense los contempla en el plan de estudios de su carrera.

- Se logró reorganizar el plan de estudios, incluyendo una visión humanística, menos técnica y una justa valoración de los cursos.

- Se logró un programa más equilibrado e integrado con una visión de la ingeniería bastante integral, no tan especializado, sino generalista.

- Se crearon estrategias de ordenamiento de la información, así como el acceso rápido y actualizado a ésta.

- Se propiciaron prácticas de sistematización de la información y de las experiencias (Quirós, Arce, y Rojas, 2003, p. 53)

\subsection{Limitaciones de la equivalencia sustancial con el CEAB}

Las limitaciones se deben entender como aquellas situaciones durante y después de la "equivalencia" que impliquen aspectos negativos y contradictorios a los programas de ingeniería que han optado por este sistema. En ese sentido, tienen una gran importancia, pues les indican a las universidades y al CFIA, los aspectos en los que deben prestar mayor atención, en cuanto a los criterios evaluativos seleccionados.

Una de las limitaciones es el hecho de que las licenciaturas en ingeniería costarricenses, son equivalentes solamente a un bachillerato canadiense, debido a que nuestro sistema educativo cuenta con menos años de escolaridad y presenta una deficiencia en secundaria en las áreas de matemática, física y química. 
Aunque la mayoría de las personas entrevistadas, afirma que los cambios no han significado modificaciones radicales en su visión y plan de estudios, lo cierto es que, como lo establece el Ing. Olman Vargas,

Si usted acepta la acreditación con los canadienses, está aceptando que va a tener núcleos básicos, que va tener un desarrollo equilibrado de las áreas que ellos definen como fundamentales; es decir, está aceptando toda una línea de pensamiento de lo que es o debe ser un programa de ingeniería. (Arce y Quirós, 2005b)

\section{Recomendaciones}

La agencia de acreditación canadiense CEAB es una agencia cuyos objetivos, criterios y procedimientos para evaluar programas de ingeniería son coherentes y responden a las nuevas corrientes de globalización e internacionalización de las naciones. Pero antes de tomar la decisión de optar una equivalencia sustancial con esta agencia. Las autoridades administrativas y académicas de los programas de ingeniería, deben estudiar en profundidad los requisitos mínimos en todos los ejes curriculares; el instrumento de evaluación utilizado; la rigurosidad de la visita de los pares externos; la conversión de las unidades de acreditación. Lo anterior se presenta con el objetivo de contar con bases sólidas para determinar si el programa que opte por sus criterios evaluativos, cumple con los requerimientos establecidos por esta agencia. No debemos olvidar que esta agencia es la encargada de la acreditación de los programas de ingeniería en Canadá y, que al evaluar programas en otros países, utiliza exactamente los mismos criterios y estándares de su país. Finalmente, es importante tener presente que los programas de ingeniería de nuestro país, obtienen no una acreditación, sino una equivalencia sustancial, en el sentido de que sus planes de estudio son "sustancialmente equivalentes" con los planes de estudio de los programas canadienses que se han acreditado por la CEAB. Sin embargo, lo interesante de este punto, es cómo en el imaginario colectivo universitario consultado para la investigación, se plantea la acreditación como un hecho, y no como lo que es, una equivalencia sustancial. 


\section{Referencias}

Arce, R., Quirós, M. (2005a). Comunicación personal. Entrevista al Ing. Ismael Mazón, Coordinador de la Comisión de Acreditación del Programa de Ingeniería Eléctrica de la Universidad de Costa Rica, realizada el 19 de julio del 2002.

Arce, R., Quirós, M. (2005b). Comunicación personal. Entrevista al Ing. Olman Vargas, Decano de la Facultad de Ingeniería de la Universidad Latina, realizada el 29 de julio del 2002.

Arce, R., Quirós, M. (2005c). Comunicación personal. Entrevista al Ing. Fernando Silesky, Decano de la Facultad de Ingeniería de la Universidad de Costa Rica, realizada el 15 de octubre del 2002.

Arce, R., Quirós, M. (2005d). Comunicación personal. Entrevista al Ing. Roberto Trejos, Presidente de la Comisión de Acreditación del Colegio Federado de Ingenieros y Arquitectos, realizada el 24 de julio del 2002.

Arce, R., Quirós, M. y Rojas, O. (2003). Contextualización de los sistemas de acreditación internacional: el caso de la agencia canadiense CCPE-CEAB en las universidades costarricenses. San José, Costa Rica: Documento no publicado.

Bernal, A. (1999). Los sistemas educativos canadienses y españoles. Toronto: Colegio Bishop Strachan.

CCPE-CEAB. (1998). Acreditation Criteria and Procedures. Traducción del M.A Oscar Rojas. Canadá: CCPE-CEAB. Disponible en www.ccpe.ca

CIEES (1994). Comité de Ingeniería y Tecnología: marco de referencia para la evaluación. México: Coordinación Nacional para la Planeación de la Educación Superior.

Colegio Federado de Ingenieros y Arquitectos. (s.f.). Criterios de acreditación. San José, Costa Rica: Documento borrador de la Comisión de Acreditación del CFIA.

Coto, J. (1997). Acreditación a paso firme. En Boletín Informativo. Número: 84. Setiembre y Octubre.

Fallas, J. y Cox, A. (2002). Estudio de empleadores de los profesionales en ingeniería en Costa Rica, 2001: Informe final. San José: CONARE, OPES.

Gómez, A. (2001). ¿Es necesaria la certificación profesional? México, Hemeroteca Virtual ANUIES. Disponible en http://www.hemerodigital.unam.mx/ANUIES.

Instituto Colombiano para el Fomento de la Educación Superior (1994). Simposio sobre acreditación universitaria. Colombia: El Instituto.

Municio, P. (1997). Herramientas para la evaluación de la calidad. Barcelona: CISSPRAXIS Educación. 
REDICA-CSUCA. (s.f.). Propuesta de criterios y estándares para la acreditación de las carreras de ingeniería en el nivel de Centro América, Panamá y Belice. Documento de trabajo. 